\title{
An Improved Variogram Analysis of the Maximum Expected Disparity in Stereo Images
}

\author{
Bogusław Cyganek and Jan Borgosz \\ University of Mining and Metallurgy \\ Department of Electronics \\ Al. Mickiewicza 30, 30-059 Kraków, Poland \\ \{cyganek, borgosz\}@uci.agh.edu.pl
}

\begin{abstract}
This paper concerns the method of estimation of one of the initial parameters for stereo image processing - the maximum expected disparity value. An automatic assessment of this parameter would benefit in improvement of automation and performance of stereo methods. The authors have developed an improved version of the heuristic method of estimation of the maximum disparity value for real stereo images. It is based on statistical analysis of the spatial correlation between stereo images - the so called image variograms, used so far only to single images and extended to the processing of stereo views by authors of this paper.
\end{abstract}

\section{Introduction}

In the stereo processing an a priori knowledge of the maximum disparity value is necessary to set a proper range of iteration. The accurate choice of the maximum disparity threshold value for almost any known stereo processing method [8] is crucial to the quality of the output disparity map and to the computation time [2][5]. A too small value of this threshold - comparing to the true maximum disparity that will be encountered in images - leads to false disparity map with many errors. On the contrary, the too big disparity range produces many errors as well, because of significant amount of false matches. Furthermore, selection of the disparity range should adapt to the true distance to the matched object. Considering the computation load, the smaller values for this parameter give shorter calculations time [1]. Therefore an initial choice of this parameter should be as close as possible to the true maximum disparity expected in the input images.

Although usually this parameter is set somehow arbitrarily, the automatic method would be advantageous in many real image processing systems. One example can pose a vision system which navigates blind person in real word developed also by authors [4].

Solution to the estimation of the maximum disparity value were investigated by many researchers [1][2][7][9]. Methods presented in listed sources may be classified as a deterministic approaches. An opposite is a statistical approach which will be explored in this paper. 
In this work we present a heuristic solution to the problem of automatic and $a$ priori estimation of a maximum disparity threshold for real stereo images. The presented method is an improved version of the method presented in [3]. Also in this new approach we rely on the statistic method - the so called image variograms, used so far only for analysis of single images and extended to the processing of stereo views by the authors. Many experiments were performed to affirm this method, as well as a comparison of the results obtained with this heuristic approach to the much more ideal disparity estimation made by humans [2].

\section{Variogram Analysis for Stereo Images}

Finding a disparity between two images can be accomplished by an examination of the spatial structure of a both images - this can be stated in a form of an analysis of so called a regionalized variable [10]. One way to do this is to analytically relate the change of this variable in a form of a function of some separating distance $h$. The solution to this problem would be in a form of a variogram, which defines a spatial correlation or structure of a regionalized function and is given by [6][10]:

$$
\gamma(\mathrm{h})=\frac{1}{2} \operatorname{Var}(f(x+h)-f(x))
$$

where $\operatorname{Var}()$ is the variance function, $f(x)$ is an image signal function and $h$ the separating distance. Shape of the variogram reflects the degree of correlation between samples of images. Variogram functions that rise as $h$ increases indicates that the spatial correlation decreases as more distant samples are chosen, until a separation distance is reached at which knowledge of one sample tells us nothing about another ones [10]. For two dimensional signals - such as images - the variogram was defined by Ramstein as follows:

$$
\gamma(\mathrm{h})=\frac{1}{2 \mathrm{~N}_{\mathrm{c}} \mathrm{N}_{\mathrm{r}}} \cdot\left[\sum_{x=1}^{N_{c}} \sum_{y=1}^{N_{l}}[f(x+h, y)-f(x, y)]^{2}-\sum_{x=1}^{N_{c}} \sum_{y=1}^{N_{l}}[f(x, y+h)-f(x, y)]^{2}\right]
$$

where: $N_{c}$ and $N_{r}$ are respectively the number of rows and columns of the image, and $h$ is a distance expressed in pixels [6].

In this paper we propose a modification to the equation (2) - we define variograms for the two images (stereo pair) as follows:

$$
\gamma(\mathrm{h})=\frac{1}{2 \mathrm{~N}_{\mathrm{c}} \mathrm{N}_{\mathrm{r}}} \sum_{x=1}^{N_{c}} \sum_{y=1}^{N_{r}}\left[f_{l}(x+h, y)-f_{r}(x, y)\right]^{2}
$$

where: $f_{l}$ and $f_{r}$ are the left and right pictures from the stereo pair, respectively. 


\section{An Heuristic Estimation of the Maximum Expected Disparity}

The statistical analysis of stereo images with variograms relies on creation and interpretation of variograms given by (3) for different values of the special argument $h$. There are many theoretical models of variograms and rules set by statistician for their analysis [10]. One of the most prominent test is to check behavior of a variogram at the origin - the measure that tells more less on spatial correlation of a signal.

To find a similar rule for stereo images the authors have performed the variogram analysis of many different stereo images. Fig. 1 shows some of the representing stereo images - images no 1 and 2 are characteristic by large, while 3 and 4 small, values of a maximum disparity.

1
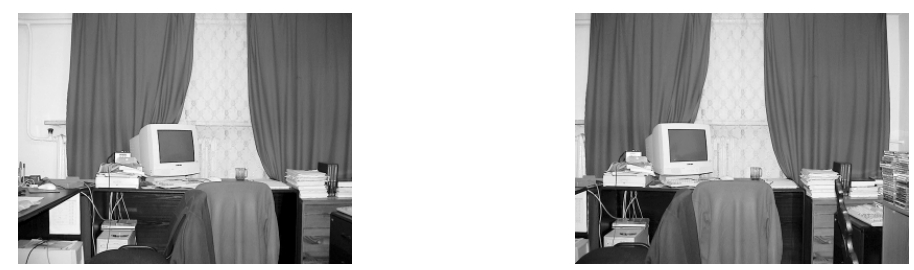

2
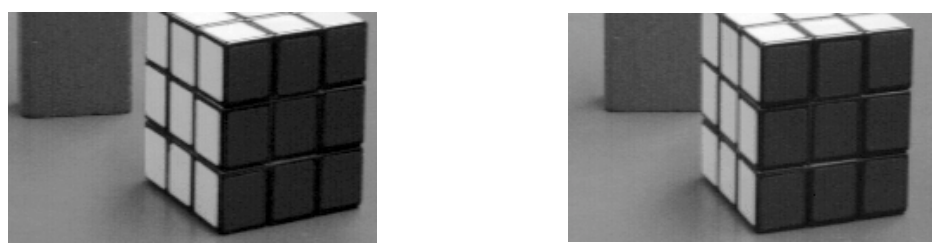

3
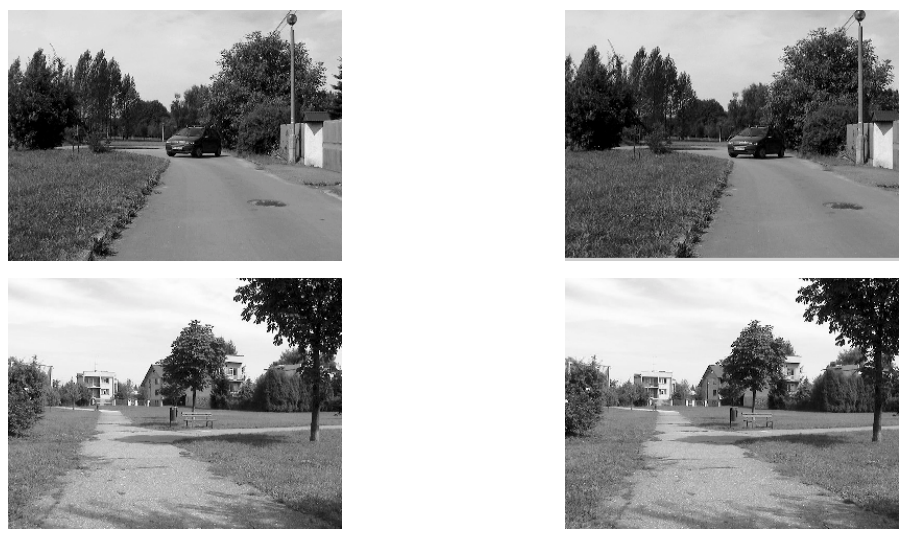

4

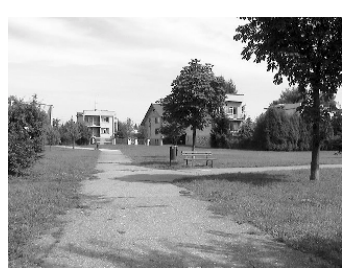

Fig. 1. Representative stereo images used for variogram analysis: 1) Office, 2) Rubic (CIL CMU), 3) Punto, 4) Park (1, 3, and 4 - University of Mining and Metallurgy). Images 1) and 2) represent a group of images with large disparity, 3) and 4) with small maximum disparity

Based on the variogram analysis it appeared that the most prominent feature differentiating stereo images is a behavior of a corresponding variogram at origin and a type of a first local minimum in this variogram. 
Two identical images ( 0 disparity)
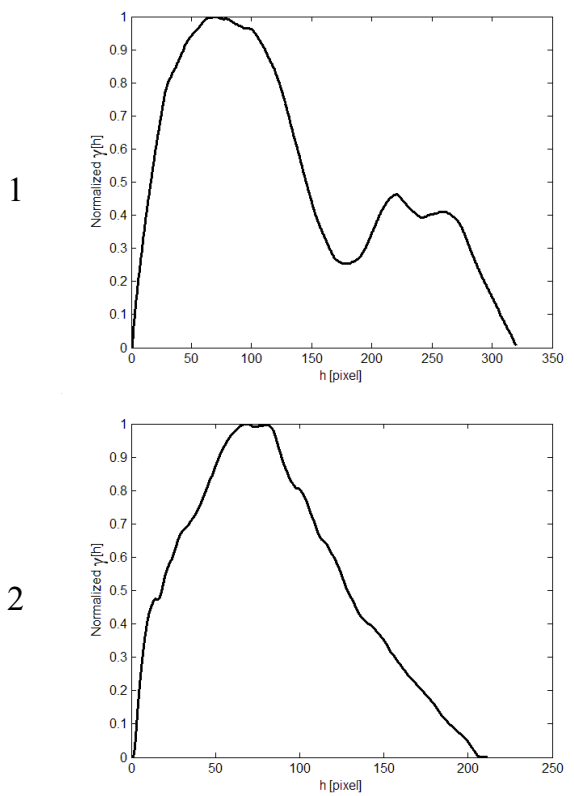

3
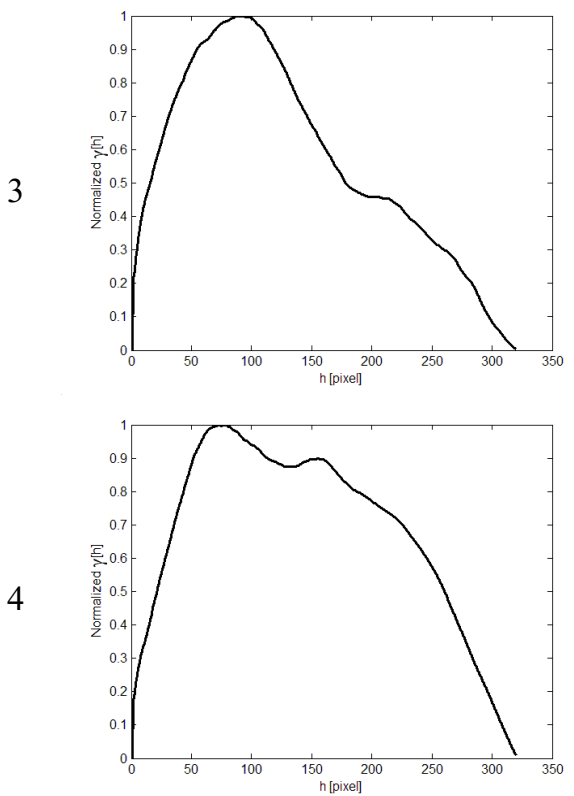

Two images of a stereo pair
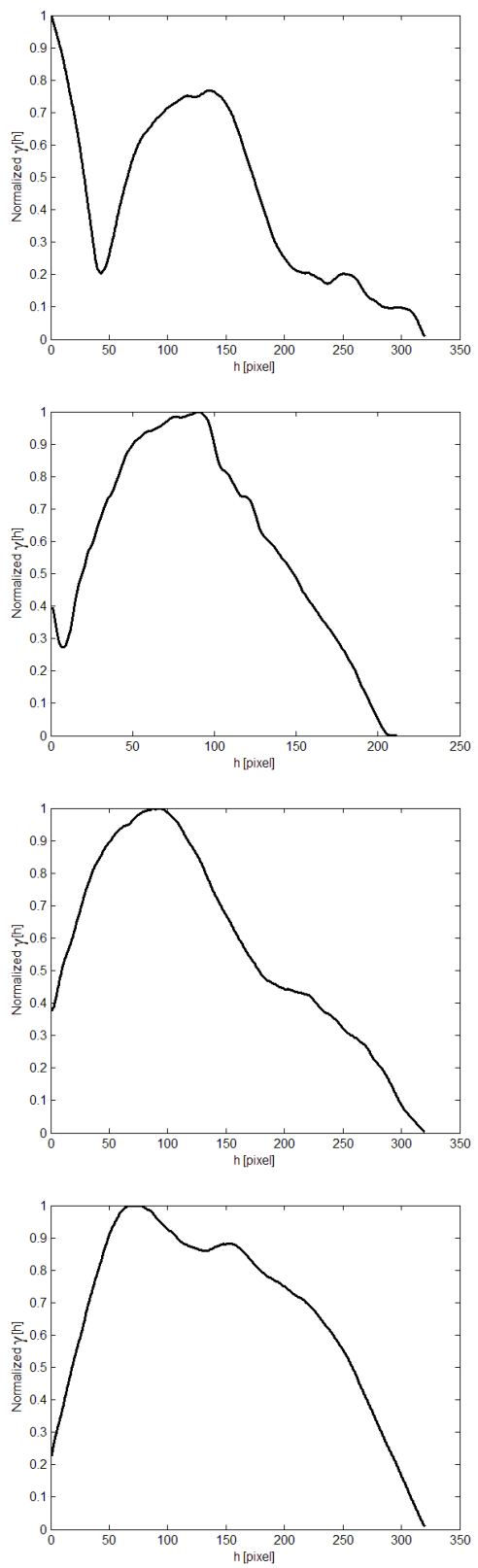

Fig. 2. Variograms for corresponding images of Fig. 1

Fig. 2 depicts two classes of variograms performed on images from the Fig. 1: 
- The variograms from the first column were obtained for two identical (left) images of each stereo pair;

- The second column contains variograms for each stereo pair.

Based on a scrutiny of over a fifty of different stereo images, it appears that considering the variogram analysis, most of the real stereo images could be divided into those that have significant amount of close objects - and thus expose large values of a maximum expected disparity - and those that are too "similar", or in other words, those that do not have a sufficient amount of close objects that would contribute to a significant maximum disparity. Exemplary images 1 and 2 in Fig. 1. belong to the first group, while 3 and 4 to the second one.

Based on the aforementioned observations the author tried to set a heuristic rule for determination of a maximum expected disparity value in stereo images.

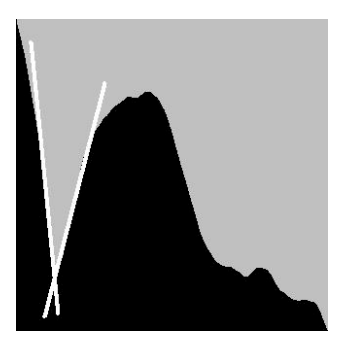

Fig. 3. Behavioral analysis of a variogram for determination of a maximum expected disparity value. There is visible a large value at the origin, and a significant local minimum that gives a starting estimate for a maximum disparity

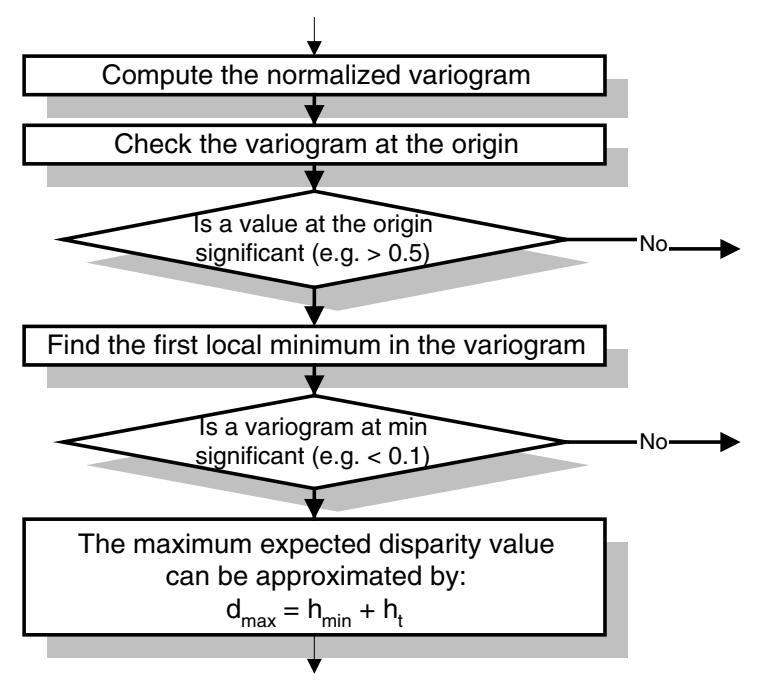

Algorithm 1. A flowchart for computation of an expected maximum disparity value in stereo images

The behavioral analysis of a variogram consists of an observation of its value at the origin, as well as on noticing a position and "strength" of a first minimum - see 
Fig. 3. Stating this it is possible to formulate the sought rule in a form of the Algorithm 1.

The computation starts with a normalized variogram. At first a variogram value at the origin is checked, and if it is much above zero, computations proceed. Then a local minimum is searched, and if it is significant, it constitutes a starting point for determination of the expected maximum disparity value $d_{\max }$, as follows:

$$
d_{\max }=h_{\min }+h_{t}
$$

where a $h_{\min }$ is a found local minimum place in the normalized variogram, $h_{t}$ stands for a threshold value, usually chosen to be between $10-50 \%$ of $h_{\min }$.

\section{Conclusions}

This paper presents a heuristic method of an a priori estimation of the expected maximum disparity values for some of stereo images. A knowledge of this input parameter is fundamental for consequent stereo matching process. The maximum disparity value, usually set in an arbitrary manner in a form of a threshold, can now be more precisely assessed by means of the presented computer algorithm.

The method was developed based on many experiments with real stereo images that led to the important partitioning rule and, in consequence, to the heuristic method stated in Sect. 3. The presented algorithm was implemented in $\mathrm{C}++$ and further tested. Those tests showed a great degree of conformity among maximum disparity values computed by the presented algorithm and those found by a testing person (which values can be assumed to be a reference).

\section{References}

1. Alvarez, L., Deriche, R., Sanchez, J., Weickert, J.: Dense Disparity Map Estimation Respecting Image Discontinuities. INRIA Technical Report No 3874 (2000)

2. Cyganek, B.: Three Dimensional Image Processing, (in Polish) EXIT Warsaw (2002)

3. Cyganek, B., Borgosz, J.: Maximum Disparity Threshold Estimation for Stereo Imaging Systems via Variogram Analysis. Accepted to the ICCS 03, Russia (2003)

4. Cyganek, B., Borgosz, J.: Computer Platform for Transformation of Visual Information into Sound Sensations for Vision Impaired Persons. LNCS 2626, Springer (2003) 182-191

5. Glass, C., Carr, J., Yang, H.M., Myers, D.: Application of spatial statistics to analyzing multiple remote sensing data set, Geotechnical Applications of Remote Sensing and Remote Data Transmission, ASTM, Philadelphia (1987) 139 - 150

6. Ramstein, G., Raffy, M.: Analysis of the structure if radiometric remotely - sensed images, International Journal Of Remote Snesing, 10 (1989) 1049 - 1073

7. Scharstein, D.: View Synthesis Using Stereo Vision. LNCS 1582, Springer-Verlag (1999)

8. Scharstein, D., Szeliski, R.: A Taxonomy and Evaluation of Dense Two-Frame Stereo Correspondence Algorithms. IJCV, Vol. 47,1 No. 1-3 (2002) 7-42

9. Sporring, J., Nielsen, M., Florack, L., Johansen, P.: Gaussian Scale-Space Theory. Kluwer Academic Publishers (1997)

10. Starck, J.-L., Murtagh, F., Bijaoui, A.: Image Processing and Data Analysis. The Multiscale Approach. Cambridge University Press (2000) 